\title{
La réactivation des Routes de la soie par la Chine : une nouvelle marginalisation de l'Europe
}

\author{
Aurélie Lacassagne \\ Université Laurentienne, Canada
}

\begin{abstract}
Résumé : Les Routes de la soie historiques qui ont relié l'Orient et l'Occident pendant des siècles ont de tout temps revêtu des dimensions politiques, économiques, et culturelles fondamentales. Il est intéressant d'assister à la réactivation de ces routes, par la Chine notamment, au moment même où les héritiers des trois autres empires historiques de ces routes (ottoman, perse, et russe) ressurgissent sous de nouvelles formes et s'inscrivent dans une histoire sur la longue durée. On peut se poser la question de savoir si cette réémergence des Routes de la soie contribuera à un équilibre des puissances producteur de paix ou se révèlera un élément de perturbation de plus dans un monde déjà en profonde reconfiguration. Une chose semble avérer : l'existence passée et présente des routes de la soie acte la position périphérique qu'occupe l'Europe dans l'espace eurasiatique. Dans un premier temps nous reviendrons sur les imaginaires liés aux routes de la soie afin de mettre en contexte le rôle finalement marginal que l'Europe a pu jouer. Dans un deuxième temps, nous verrons comment les routes actuelles contribuent également à une périphérisation de l'Europe.
\end{abstract}

Mots clé : Route de la soie, Chine, Europe, espace eurasiatique, géopolitique, marginalisation

Abstract: The historic Silk Roads, which have connected East and West for centuries, have always had fundamental political, economic, and cultural dimensions. It is interesting to witness the reactivation of these roads, especially by China, just as the heirs of the three other historic empires of these roads (Ottoman, Persian, and Russian) are resurging in new forms and as part of a longer history. The question is whether this re-emergence of the Silk Roads will contribute to a balance of peace-producing powers or prove to be an element of further disturbance in a world already undergoing deep reconfiguration. One thing seems evident: the past and present existence of the Silk Roads act in a peripheral position occupied by Europe in the Eurasian space. As a first step, we will return to the imaginaries related to the Silk Roads in order to contextualize the ultimately marginal role that Europe could play. Then, we will see how the current roads also contribute to a peripheralization of Europe.

Keywords: Silk Road, China, Europe, Eurasian space, geopolitics, marginalization

\section{Introduction}

"Comme la vie me laisse très peu de chances de sortir librement, les routes sont pour moi un pont précieux vers des mondes inconnus » Empereur Naruhito (Schmidt, 2019).

Aurélie Lacassagne est professeure agrégée dans le Département de science politique à Université Laurentienne. Courriel : alacassagne@laurentienne.ca

Culture and Local Governance / Culture et gouvernance locale, vol. 6, no. 1, 2019. ISSN 1911-7469 Centre on Governance, University of Ottawa, 120 university, Ottawa, Ontario, Canada K1N 6N5 
Les Routes de la soie historiques qui ont relié l'Orient et l'Occident pendant des siècles ont de tout temps revêtu des dimensions politiques, économiques, symboliques et culturelles fondamentales. Il est intéressant d'assister à la réactivation de ces routes, par la Chine notamment, au moment même où les héritiers des trois autres empires historiques de ces routes (ottoman, perse, et russe) ressurgissent sous de nouvelles formes et s'inscrivent dans une histoire sur la longue durée. Entre l'obsession chinoise de contrôler des routes mondiales d'approvisionnement pour nourrir sa population ; le pantouranisme comme vecteur de la politique étrangère turque ; I'influence tenace du (néo-)eurasisme en Russie ; et la position assumée de chef de file du monde chiite par l'Iran, il semblerait que ces routes, autrefois pivots géopolitiques d'un certain équilibre du monde, reviennent sur le devant de la scène. On peut néanmoins se poser la question de savoir si cette réémergence des Routes de la soie contribuera à un équilibre des puissances producteur de paix ou se révèlera un élément de perturbation de plus dans un monde déjà en profonde reconfiguration. Une chose semble avérer : l'existence passée et présente des routes de la soie acte la position périphérique qu'occupe l'Europe dans l'espace eurasiatique. Dans un premier temps nous reviendrons sur les imaginaires liés aux routes de la soie afin de mettre en contexte le rôle finalement marginal que l'Europe a pu jouer. Dans un deuxième temps, nous verrons comment les routes actuelles contribuent également à une périphérisation et à une provincialisation (Chakrabarty, 2000) de l'Europe.

\section{Imaginaires des Routes de la soie sur la longue durée}

La Route de la soie est une notion « forgée par le géographe allemand Ferdinand von Richtofen, en 1877, pour désigner le réseau d'itinéraires empruntés par les marchands entre la Chine et la Méditerranée pendant l'Antiquité et le Moyen Âge " (Thorez, 2016, p. 299). Certains critiquent le romantisme dont elle a fait l'objet (Rezakhani, 2010, p. 420). Ils expliquent que s'est développé un grand récit s'agençant autour d'images de déserts, de villes exotiques, d'oasis perdus tout en reconnaissant que "les régions de la Transoxiane, des monts Pamir, et du bassin du Turkestan/Tarim sont des sources importantes pour l'étude de l'histoire mondiale, et leur étude est centrale pour comprendre la progression de l'histoire et des relations historiques " (Rezakhani, 2010, p. 420). Ces directions, représentations, descriptions font l'objet d'intenses discours de la part d'une multitude d'acteurs à travers les siècles ce qu'illustre bien son importance discursive et mémorielle. Au final, peu importe qu'elle ait existé, qu'il y en ait plusieurs, que son trajet soit disputé, elle est un construit social ayant des conséquences, elles, bien réelles.

\section{Des routes de la soie constitutives d'un espace eurasiatique}

On ne peut nier que les routes de la soie occupent une place à part dans l'imaginaire de nombreux peuples étant donné qu'elles s'inscrivent tout autant qu'elles ont produit plusieurs « constellations civilisationnelles " (Delanty, 2003, p.15) qui se sont constituées mutuellement en grande partie au travers de ces routes. D'ailleurs, on pourrait avancer que l'existence multiséculaire et la marque 
profonde de ces routes sur la culture, les langues, habitus, économies, politiques, histoires et sociétés créent en quelque sorte une "sphère civilisationnelle " (Arnason, 2000, p. 44) commune : un espace eurasiatique.

Au cœur des routes de la soie l'on retrouve donc l'Asie centrale, le " cœur même du monde (Heartland) » (Lindley, 2009, p. 115). La maitrise de " ce pivot géographique de l'histoire ", selon Mackinder (1904, p. 421-437), assurerait le contrôle du monde. Malgré les nombreuses critiques émises à l'encontre de cette approche géopolitique déterministe, il n'en demeure pas moins que cet espace eurasiatique semble encore aujourd'hui constituer un centre important pour l'équilibre du monde. L'Eurasie est depuis des siècles cet immense espace qui part de la Chine englobe l'espace russe, l'Asie centrale, le Caucase, les anciens territoires persan et ottoman pour atteindre la Méditerranée et ses pays côtiers, jusqu'à l'Europe. II rejoint la mer Baltique via l'isthme mer Noiremer Baltique et l'océan arctique via l'immensité russe. Ces différents espaces ont permis les échanges, non seulement économiques mais aussi culturels et scientifiques. Ils ont également permis le brassage des peuples et leurs savoirs si bien qu'il est souvent ardu de trouver une origine. Il y aurait là un parallèle intéressant à faire avec l'espace caribéen créolisé tel que décrit par Glissant (1990).

Ce sont en partie ces routes de la Soie que les Mongols empruntent pour construire ce qui demeure à date le plus grand empire n'ayant jamais existé. Quelques siècles auparavant, Alexandre le Grand construira son Empire en suivant des traces analogues. La forme politique même d'Empire prend naissance dans ces confins eurasiatiques des Routes de la soie comme le rappelle Bruneau (2018, p. 3). Que ce soit la Mésopotamie, l'empire perse, l'empire grec d'Alexandre, les empires arabo-musulmans, l'empire des Han, l'empire ottoman, l'empire romain, jusqu'à l'empire mongol. C'est dans le contexte de ces empires que les routes de la soie, comme espaces de circulation, surgissent dans les deux premiers siècles de notre ère. Jusqu'au $20^{\mathrm{e}}$ siècle, l'espace eurasiatique est marqué par la présence de ces nombreux empires. Il s'agit là d'un fait important à plusieurs égards. Premièrement, cela permettrait d'expliquer en partie pourquoi ces territoires, depuis la désintégration forcée des Empires suivant la Première guerre mondiale, peinent à stabiliser les États nouvellement créés et surtout à installer la démocratie, système politique étranger aux mœurs politiques de ces régions (Bruneau, 2018, p. 11). Deuxièmement, cela explique les mosaïques aussi bien ethnoculturelles que religieuses ou linguistiques, complexes que l'on retrouve sur ces espaces. Ces empires ont largement fonctionné sur un modèle que l'on appellerait aujourd'hui multiculturaliste. En effet, au sein de ces empires - et contrairement aux empires coloniaux européens - il n'y a pas eu de volonté d'homogénéisation. L'empire ottoman par exemple fonctionnait sur le principe de la reconnaissance de l'autonomie des millets, qui conservaient leurs systèmes juridiques, langues et systèmes d'éducation, tout en versant une taxe au Sultan pour sa protection. L'empire mongol représente par excellence cette ouverture et tolérance envers les peuples conquis qui va même, pour nombre d'entre eux, à adopter la religion des terres sur lesquels ils se trouvent, notamment l'islam (Bruneau, 2018, p. 5). En réalité, seuls l'empire perse et l'empire chinois ont fait montre d'une certaine homogénéité même si les variations, notamment religieuses, se sont développées au fil des époques (Bruneau, 2018, p. 6). 
Cette digression nous permet de mieux comprendre la complexité de notre objet. Souvent on parle de la route de la soie, je préfère utiliser le terme au pluriel car comme le rappelle Lindley (2009):

À dire vrai, ce n'était pas une route, mais une piste, ou plutôt des pistes. Les caravanes venaient de Méditerranée et de l'ouest par la Turquie, mais aussi de Russie méridionale par les côtes septentrionales de la mer Noire ou par la Perse, au sud de la Caspienne. Depuis I'Inde, une piste montait à l'assaut de la haute vallée de I'Indus à travers le massif de Karakoram et le col de Khunjerab avant de redescendre sur la Chine. Une route moins abrupte et très prisée passait par la partie afghane du massif du Pamir à travers le "corridor" de Wakhan. Toutes ces branches différentes de la route de la soie convergeaient dans la partie turcophone de la Chine, sa province occidentale du Xinjiang. Une fois parvenues dans cette région reculée d'Asie centrale, elles se séparaient à nouveau sur le plus de mille deux cents kilomètres en longeant soit le sud, soit le nord de l'immense désert de Taklimakan - qui rejoint au nord-est le désert mongolien de Gobi - avant d'atteindre les riches marchés des villes chinoises. (p. 117)

Cette description détaillée nous permet de bien comprendre la nature profondément rhizomique des routes de la soie. Si j'emploie ici le mot rhizome, c'est bien sûr pour faire écho à ce concept de Deleuze et Guatarri (1980), qui rejoint également celui de nomadisme. Car ces peuples parcourant les routes de la soie étaient bien, pour la plupart, des nomades qui n'hésitaient nullement à se mélanger aux autres peuples, à embrasser leur religion, à adopter les us et coutumes (Linidley, 2009, p. 119).

\section{L’Europe à la périphérie des imaginaires liés aux routes de la soie}

Le long de ces routes de la soie s'égrènent des villes cosmopolites qui peuplent l'imaginaire oriental et européen. Pour chaque peuple eurasiatique, ces villes portant le nom de Xi'an (ancienne Chang'an) Samarkand, Khiva, Boukhara, Bakou, Tabriz, Téhéran, Antioche, Constantinople/Istanbul, Alexandrie, Venise (on remarquera que c'est la seule ville profondément européenne qui peuple cet imaginaire). Ces lieux mythiques font résonner des émotions, s'inscrivent dans les contes folkloriques et récits mythiques, font jaillir des images et des architectures, se rattachent à des personnes (une armée enterrée de soldats en terre cuite, Tamerlan, Gengis Khan, Marco Polo, Avicenne/Ibn Sīnā) qui révèlent l'empreinte profonde que ces routes ont laissée.

Dans l'imaginaire proprement européen, Marco Polo est le plus illustre de ces personnages. Tout le monde connaît Marco Polo même si peu de personnes ont lu les quelques six volumes constituant son ouvrage Le Devisement du monde (Ménard, 2001-2008 [1298]). Mais cet aventurier marchand opère une attraction mythique sur l'imaginaire des Européens jusqu'à aujourd'hui comme en témoigne le fait qu'une des premières séries produites par Netflix portait sur ce personnage. Il cristallise, en quelque sorte, le rapport que les Européens entretiennent avec la route de la soie, un monde inconnu qui fascine, celui des hordes mongoles, des khanats, de la cour 
impériale chinoise, du passé glorieux de Byzance, de Venise la Sérénissime, des steppes russes. Mais la fascination inverse existe elle aussi. Marco Polo relate que le Grand Khan « voulait que lui soit envoyé une centaine de savants qui puissent enseigner la foi chrétienne et faire connaître les sept arts libéraux, c'est-à-dire la base de l'enseignement occidental. Le Grand Khan avait certainement le désir de mieux connaître les Occidentaux et leur culture après son entretien avec les Polo " (Racine, 2011, p. 331). Il est impossible de penser à Marco Polo sans également mentionner Kubilaï. Petit-fils de Gengis Khan, élu Grand Khan en 1260, il établit la capitale de son empire à Pékin (nommée alors Khanbalik, ou Cambaluc) et devient Empereur de Chine en proclamant l'ère de Yuan en 1271. Preuve que ces personnages historiques animent encore l'imaginaire des Européens, une pièce de théâtre, signée Éric Bouvron, en 2017 met en scène ces deux figures dans Marco Polo et I'hirondelle du Khan, sur les planches du Théâtre La Bruyère à Paris (Tranchant et Ponthieux, 2017).

$S^{\prime}$ intéresser un peu aux tribulations de ces deux personnages nous permet de mettre en valeur les ambivalences quant à la notion d'Orient. L'Orient, tel qu'imaginé, constitué dans la tête des Occidentaux, n'est pas un bloc homogène. Au fil de l'histoire, cet Orient se voit incarner par différentes figures : le Turc, l'Arabe, le Mongol, le Chinois. Ainsi Marco Polo entreprend son voyage, soit, en espérant bien ramener des marchandises de grande valeur, mais comme le rappelle Pierre Racine (2011), il part avant tout à la cour du Grand khan « comme ambassadeur du pape » car « son père et son oncle sont revenus de la cour du Grand khan en 1269, apportant un message de Kubilai au pape » (p. 328). Grégoire X souhaite obtenir l'appui des Mongols dans son projet de croisade (Racine, 2011, p. 331). II cherche l'appui d'un certain Orient, contre un autre orient. II y a une fascination positive pour les richesses et la douceur de vie à la cour de Kubilaï (Racine, 2011, p. 335), alors qu'il n'y a que détestation, fascination morbide pour les musulmans occupant Jérusalem. La Chine continuera pendant des siècles à incarner une curiosité positive chez les Occidentaux comme en témoigne la mode des chinoiseries en Europe de la Renaissance au $18^{\mathrm{e}}$ siècle. La tendance s'inverse à la fin du $19^{e}$ siècle quand cet extrême Orient sera alors dépeint sous des traits barbares, c'est le " péril jaune ". La résistance chinoise aux visées impérialistes européennes et, plus tard, la cuisante défaite russe face au Japon en 1905, modifie l'imaginaire européen quant à cet Orient. Dans le même processus, l'Orient turc et perse au $19^{\mathrm{e}}$ siècle jouit d'un a priori plus favorable comme le renouveau de l'orientalisme dans la peinture (Ingres, etc.) et la littérature (Flaubert, etc.) européenne en témoigne. Mais bien sûr les empires ottoman et perse ne sont plus à cette époque là que l'ombre d'eux-mêmes et complètement sous la coupe des Européens, Anglais et Français en tête.

Il faut également prendre conscience que du côté de "l'Orient " ou des "Orients ", l'Europe n'occupe pas la place qu'elle pense avoir. Rezakhani (2010) soulève un point fort intéressant quand il fait remarquer que quand les Chinois parlent de l'Ouest, ils ne se réfèrent pas à Rome :

en apportant des preuves d'échanges commerciaux, les scientifiques citent page après page de chroniques chinoises sur le commerce avec l'Ouest, et ensuite ils apportent des preuves issues de sources romaines sur le commerce avec l'est. Dans le processus, ce qu'ils ignorent c'est que l'ouest et l'est en question diffèrent des 
termes culturels assignés par la science moderne pour dénoter les civilisations occidentale et orientale. Les sources chinoises, en effet, parlent de commerce, et de diplomatie, avec la Transoxiane et l'Iran, et les sources romaines se soucient de tenter de participer de quelque façon à ce commerce. (p. 431)

Les Européens manquent également de profondeur historique dans leur appréhension de ces routes. En effet, si dans l'imaginaire européen, c'est Marco Polo (et en fait son père et son oncle) qui sont considérés comme les pionniers des routes de la soie, Lindley (2009) nous rappelle que "Les récits de voyages d'ouest en est remontent en réalité beaucoup plus loin dans le temps » (p. 118). Cette remarque fort opportune nous rappelle donc que l'Europe, et que des Européens comme les Polo, occupent une place somme tout assez marginale dans les dynamiques des routes de la soie.

Cependant, l'inverse n'est pas vrai : les routes de la soie jouent un rôle prépondérant sur l'Europe. Il s'agit là d'un paradoxe, en réalité de plusieurs paradoxes, forts intéressants et que l'on pourrait résumer ainsi. Premièrement, alors que l'Europe a développé tout un imaginaire autour des Routes de la soie, elle en a toujours constitué que l'extrémité lointaine comme nous venons de l'établir. Deuxièmement, les produits arrivants grâce à ces routes (la soie, les porcelaines et les épices, mais aussi le papier et la poudre à canon) vont jouer un rôle prépondérant dans l'éclosion de la dynamique du capitalisme (Braudel, 1988), tout d'abord bénéficiant à l'économie de marché, étape nécessaire au développement du capitalisme; permettant largement l'éclosion du protestantisme grâce au papier - et à l'imprimerie - qui permet à ces idées " hérétiques " de circuler ; ouvrant la voie finalement à l'accélération de la dynamique de l'Occident (Elias, 2003), à savoir la constitution des États modernes grâce aux divers processus de monopolisation, en grande partie rendus possible grâce à la poudre à canon. Autrement dit, les idées, les arts et les biens qui s'échangent le long de ces routes entre les Perses, les Chinois, les Turcs et les Arabes arriveront finalement en Europe et permettront à cette dernière de sortir de la noirceur du Moyen-Âge pour rentrer dans la Renaissance. D'ailleurs, la centralité des routes de la soie commence à être mise à mal à partir du moment, au $16^{\mathrm{e}}$ siècle véritablement, où les pays européens, développeront leurs empires maritimes. Dans la foulée, après avoir atteint son apogée avec Soliman le Magnifique au $16^{\text {e }}$ siècle, l'empire ottoman ne cessera de décliner, processus conjoint à l'extension de l'empire russe sous I'impulsion d'Ivan IV (1533-1584), qui opère des percées importantes vers l'Asie. La Chine se repliera sur elle. Le monde arabo-musulman, après avoir connu son âge d'or, débutera son déclin.

II appert clairement que l'Europe, bien que périphérique, doive énormément aux routes de la soie mais dans le même temps, elle va mettre tout ce que lui apportent les routes au service du développement de ses empires qui seront orientés vers l'Atlantique (Afrique et Amériques) et qui du même coup vont marginaliser l'espace civilisationnel des routes de la soie et recentrer l'Europe au cœur du monde...jusqu'à tout récemment où ce grand processus s'inverse.

Cette inversion, ce retournement du monde, cette grande transformation, l'Europe semble avoir du mal à en saisir les tenants et les aboutissants. En effet, jusqu'à aujourd'hui, l'Europe demeure un espace civilisationnel atlantique, qui s'est désaxé du centre méditerranéen médiéval. Se faisant, elle a, dans sa Weltanschauung, opéré une périphérisation de son flanc est, de l'espace 
méditerranéen. Malgré les faiblesses évidentes de l'arc atlantique aujourd'hui comme espace maritime (comparé au Pacifique), et malgré le caractère profondément asymétrique des relations entre l'Europe et les États-Unis, cette dernière demeure enfermée dans cette vision du monde. En partie parce qu'elle n'a pas su prendre acte des bouleversements géopolitiques provoqués par la fin de la Guerre Froide, et en partie parce qu'elle n'a pas encore accepté que la fin de ses empires coloniaux fait en sorte qu'elle n'est plus le centre du monde. L'Europe, ses dirigeants comme ses penseurs, semblent donc avoir du mal à s'intéresser à la notion d'Eurasie et la réactivation de cet espace notamment au travers de deux phénomènes : l'importance fondamentale du néo-eurasisme (Laruelle, 2001) dans la politique russe, incarné notamment par " l'Union économique eurasiatique (UEE) lancée en 2011 » et le lancement par la Chine du " projet 'One Belt One Road' (OBOR) des nouvelles 'routes de la soie' continentale et maritime, formulé, surtout à partir de 2013 " (Bruneau, 2018 , p. 1). Ce manque d'attention (ou de vision) de la part des Européens n'est pas étranger à leurs refus obstiné d'accepter la Turquie dans leurs rangs, ce qui a contribué à ce que la politique multivectorielle de la Turquie privilégie justement, une approche eurasiatique, que l'on retrouve souvent sous l'appellation de projet pantouranien.

\section{Dynamiques des nouvelles Routes de la soie : Re-périphérisation de l'Europe et recentrement du cœur de l'Eurasie}

Depuis quelques temps, les nouvelles routes de la soie font l'objet d'une grande attention de la part des médias comme des compagnies et des chancelleries à travers le monde. On peut en réalité dater un peu plus précisément la réactivation de ce vocable. Ainsi que Thorez (2016) le souligne :

Le recours à l'expression " Route de la soie " s'est généralisé dès l'accession des républiques centrasiatiques à l'indépendance pour dépeindre l'établissement de nouvelles relations politiques et surtout économiques au cœur du continent asiatique, alors que, sous l'impulsion de l'UNESCO, son emploi s'était déjà imposé à la fin des années 1980, dans le domaine culturel et scientifique, à propos des périodes anciennes [...]. L'ouverture des frontières et l'essor de nouveaux flux ont été rapidement présentés comme une renaissance de la "Route de la soie ", par contraste avec l'isolement relatif qu'avait connu la région pendant les périodes tsariste et soviétique. (p. 299)

Thorez (2016) fait remarquer également qu'un nombre impressionnant de pays s'est en réalité réapproprié « cette rhétorique de la "Route de la soie » que ce soit les pays européens, les ÉtatsUnis, « I'Inde, I'Iran, la Turquie, de même que la Corée du Sud et le Japon » (p. 302). II semble donc bien que nous assistions au renouveau du " Grand Jeu » et que tout le monde veuille être de la partie. Dans un premier temps, nous présenterons un bref aperçu de la place qu'occupent les routes de la soie dans la politique de quatre États pivots de la région : la Chine, la Turquie, l'Iran et la Russie. Ces développements ont bien sûr des impacts directs sur les cinq pays d'Asie centrale dont nous parlerons au fil de cet examen concis. Dans un deuxième temps, nous analyserons comment ce 
« Grand Jeu » peut s'apparenter à une nouvelle grande alliance eurasiatique dont l’Europe émerge comme la principale exclue.

\section{Un nouveau « Grand Jeu »}

En 2013, la Chine, par la voix de Xi Jinping, lançait un immense programme d'infrastructures visant à développer des corridors de transport reprenant ainsi " à son compte la rhétorique de la " Route de la soie ", dans le cadre de la redéfinition de sa stratégie économique et géopolitique eurasiatique " (Thorez, 2016, p. 302). Ce projet baptisé initialement One Belt, One Road (OBOR), yi dai yi lu en mandarin, s'appelle depuis 2017 le Belt and Road Initiative (BRI). II reprend ainsi de façon terrestre le chemin des routes de la soie anciennes mais se double d'une route de la soie maritime, de chemins de fer, d'oléoducs et de gazoducs. Autrement dit, nous sommes très loin de l'imaginaire des caravansaraïs. Cependant ce projet chinois, s'il s'inscrit bien dans une histoire millénaire, demeure néanmoins exceptionnel dans la mesure où il combine la voie terrestre et celle maritime (Bruneau, 2018, p. 15). Il est intéressant ici de souligner une certaine circularité. En effet, historiquement, avant que ne soit établie la route de la soie terrestre traditionnelle « qui deviendra une route largement fréquentée par les marchands italiens dans la première moitié du XIVe siècle. [...], la soie venait de Chine en grande partie par la route maritime de l'océan Indien, de la Chine méridionale jusqu'au Golfe Persique, avant de gagner par la mer Noire par la terre " (Racine, 2011, p. 320). Une chose semble avérer, si l'on se réfère à la carte ci-dessous les ambitions chinoises sont énormes. D'autant que la Chine investit également pour relier ces routes à d'autres points de transit comme Dakar, afin d'accéder à l'espace atlantique. En d'autres termes, si l'on parle de routes de la soie chinoises, il est alors facile d'oublier que ce projet de nature mondiale va bien au-delà des routes. II vise ni plus ni moins à faire de la Chine, la grande superpuissance de demain.

La Chine a peut-être saisi que le seul contrôle du Heartland est en réalité insuffisant pour contrôler le monde - l'aventure soviétique l'a démontré. II faut également maitriser les routes maritimes. Il s'agit là d'une bonne illustration des limites des routes de la soie qui sont des itinéraires terrestres même si effectivement elles sont reliées à des espaces maritimes. Dans le même temps, il est évident que pour la Chine, cette arrière-cour que représentent les routes de la soie, lui permet de sortir de la périphérie et de s'inscrire au cœur du monde. 


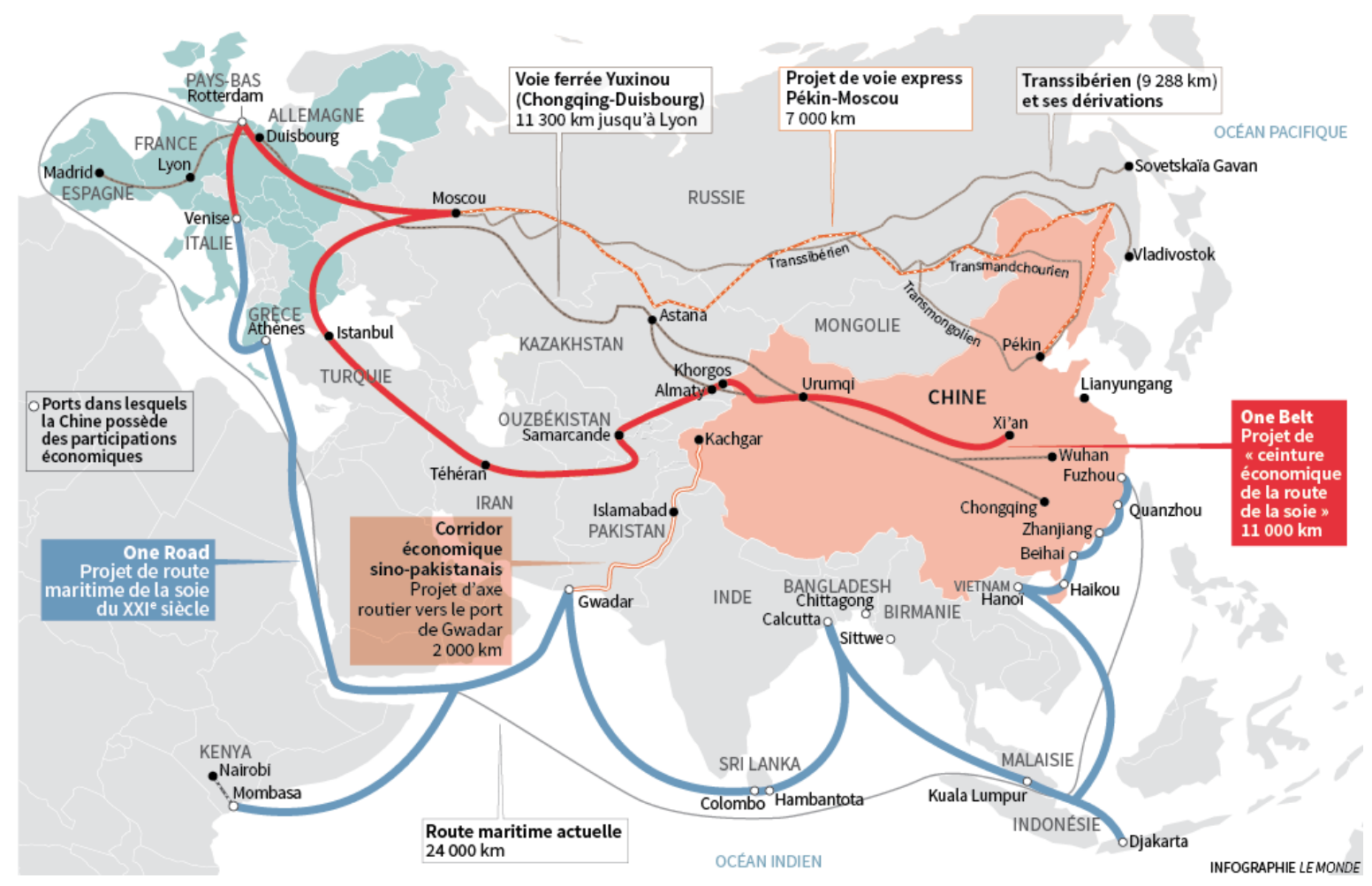

Figure 1 : Les nouvelles routes de la soie

Source : Le Monde. (2017, 13 mai). One Belt, One Road : les nouvelles routes de la soie. Repéré à https://www.lemonde.fr/economie/visuel/2017/05/13/one-belt-one-road-les-nouvelles-routes-de-lasoie_5127192_3234.html

À l'autre extrémité de la Chine, au bout de la route, on retrouve la Turquie, pont entre l'Orient et l'Europe. Elle a de tout temps occupé une position privilégiée dans l'espace eurasiatique. Son positionnement interstitiel ne facilite pas l'élaboration d'une politique étrangère simple. En réalité, qu'il s'agisse de l'Empire ottoman ou de la Turquie moderne, une politique multivectorielle s'est toujours avérée un choix sage - si ce n'est une obligation -, étant donné les nombreuses forces centrifuges et centripètes s'exerçant sur la Turquie. Depuis une quinzaine d'années et l'échec avéré de la candidature turque à l'Union européenne, la politique étrangère turque s'est clairement orientée vers l'espace eurasiatique. Qualifiée de néo-ottomaniste, cette approche repose sur l'idée de bon voisinage et s'oriente vers le monde arabe, certains pays africains, mais surtout, pour notre étude ici, vers le Caucase et l'Asie centrale - deux espaces largement turcophones. Dans les années 1990, sous la présidence de Turgut Özal, l'intérêt pour ce projet pantouranien était très important. C'était l'époque d'un nouveau grand jeu autour des ressources pétrolières et gazières de la Mer Caspienne principalement, et la Turquie voulait se placer stratégiquement dans ce jeu. Elle en a bien tiré son épingle puisqu'elle a obtenu - grâce au soutien américain, la construction du BTC (Bakou, Tbilissi, Ceyhan) qui de facto mettait hors jeu l'Iran (I'autre puissance régionale rivale) et affaiblissait la main mise de la Russie dans la région. En ce qui concerne I'Asie centrale, trois dimensions 
coexistaient en cette fin de siècle. Politiquement, la Turquie voulait se projeter (et était encouragée en ce sens) comme un modèle de démocratie pour les nouveaux États postsoviétiques (Fidan, 2010, p. 113-114; Köstem, 2017, p. 730). Économiquement, la Turquie souhaitait développer ses relations économiques en jouant la carte turcophone. Elle créa à cet effet en 1992 la TIKA (Turkish Cooperation and Development Agency). Enfin elle a voulu renforcer ses liens culturels comme en témoigne l'établissement en 1993 de la TÜRKSOY, l'Organisation internationale pour la culture turque. L'arrivée de l'AKP au pouvoir n'a pas modifié ce vecteur de la politique étrangère turque, on pourrait même en fait parler d'une institutionnalisation des relations entre les pays turcophones. Des sommets se tiennent régulièrement et en 2009 le Traité de Nakhitchevan a créé le Conseil de coopération des États turcophones (Köstem, 2017, p. 734). Cette «identité transnationale turcophone " selon l'expression de Köstem (2017, p. 729), est très utile à Erdogan dans la mesure où elle lui permet de se distancier du kémalisme qui interdisait toute forme de pan-turquisme et qui était orienté exclusivement vers l'Europe. Par ailleurs, ce pantouranisme, loin d'être aussi radical que sa conception racialiste et politique de ses origines, se déploie comme une sorte de soft power, qui ne remet pas fondamentalement en cause l'influence russe dans cet espace. De la même manière elle permet à la Turquie de se rapprocher et de développer des liens économiques avec la Chine, tout en n'interférant aucunement politiquement, Erdogan demeurant relativement silencieux quant au problème ouïgour.

Un des rivaux turcs dans la région est l'Iran. Ce pays possède une longue histoire avec les routes de la soie, elle en fut son point central comme en témoigne la circulation de la langue perse, langue des élites de toute la région, langue officielle de l'Empire mongol. Aujourd'hui, I'Iran se projette comme le héraut du monde chiite et l'on semble de préoccuper plus de ses actions sur son flanc occidental, oubliant par là même les intérêts iraniens à l'est, et son poids culturel - ne serait-ce que par les différents dialectes persans véhiculaires qui ont si bien résisté au temps et aux changements. L'Iran s'intéresse notamment de près au Tadjikistan. En effet, "L'Iran fit son terrain de jeu du rocailleux Tadjikistan, frontalier de la Chine, autrefois pointe extrême de l'Empire perse. Ici, la population est principalement chiite, incluant une forte proportion d'ismaéliens. La langue tadjik est le farsi, comme en Iran " (Lindley, 2009, p. 127). II ne faudrait pas non plus oublier les quelques huit millions d'Hazaras vivant en Afghanistan, qui après avoir joué un rôle prépondérant pendant les guerres des quatre dernières décennies, ont réussi à faire leur place dans les institutions politiques du pays. Les autorités iraniennes surveillent également leurs voisins azerbaïdjanais (la plupart chiites biens que turcophones), de même que leurs cousins kurdes (avec lesquels les Iraniens sont apparentés culturellement et linguistiquement mais opposés religieusement, les Kurdes étant sunnites). Le grand Kurdistan occupe une place majeure dans l'histoire des routes de la soie. Les velléités indépendantistes (ou autonomistes) des Kurdes habitant la Turquie et l'Irak sont une source de soucis pour le régime des Mollahs qui a déjà eu maille à partir avec les Kurdes du Rojhelat (le Kurdistan iranien) que les puissances étrangères ont toujours tenté d'amadouer pour déstabiliser le pays, pensons à l'URSS en 1946 avec l'éphémère République de Mahabad. Étant donné les sanctions américaines visant l'Iran, et dont l'objectif est d'asphyxier le pays économiquement afin de provoquer un renversement de régime, il est impératif pour Téhéran de diversifier ses marchés 
avec des partenaires qui ont les reins assez solides pour tenir tête à Washington (contrairement aux Européens). Dans ce contexte, la Chine représente un allié majeur. Cette dernière a déjà financé dans le cadre de la BRI, un chemin de fer reliant le Kazakhstan à l'Iran afin de stimuler le transport de marchandises (Farchy, 2016). Dans le cadre du BRI, la Chine a aussi développé un réseau d'oléoducs et de gazoducs avec les pays d'Asie centrale, jusqu'à la région frontalière de Sarakhs où I'Iran et le Turkménistan sont partenaires. Mais l'Iran aurait tout a gagné à développer la connexion de son réseau vers cette piste ancienne de la route de la soie.

Pour la Russie, les actions de ceux qui sont dorénavant des alliés stratégiques (Chine, Turquie et Iran) peuvent s'avérer inquiétantes dans la mesure où les pays d'Asie centrale font partie de son Étranger proche (i.e. les anciennes républiques soviétiques considérées comme zone d'influence exclusive). Le risque est grand pour elle d'être marginalisée de cette région sans laquelle elle ne peut prétendre au statut de superpuissance mondiale. Thorez (2016) résume ainsi cette quadrature du cercle pour Moscou :

Bien qu'engagée dans de nombreux programmes avec la Chine, notamment dans le cadre de l'Organisation de coopération de Shanghai, la Russie appréhende avec davantage de réserve cette notion. D'une part, la renaissance de la « Route de la soie " est souvent perçue par la Russie comme un instrument visant à la marginaliser, sur les plans politique, économique et géographique. II est vrai que de nombreux corridors de transport développés dans ce cadre, en particulier ceux soutenus par les pays occidentaux comme le programme TRACECA, évitent le territoire de la Russie. D'autre part, la Russie convoque d'autres concepts pour justifier son implication dans les affaires centrasiatiques, au premier rang desquelles la notion d'Eurasie. (p. 302-3)

En effet, depuis l'arrivée de Poutine au pouvoir, la Russie a opéré un grand tournant idéologique dans la manière de se percevoir. Suite au grand débat entre les Slavophiles et les Occidentalistes au 19e siècle, dans les années 1920 a émergé une nouvelle pensée, celle de l'eurasisme. Courant philosophique et idéologique peu connu en Occident si ce n'est à travers les travaux tardivement traduits du grand historien Lev Gumilev, le fis d'Anna Akhmatova et Nicolaï Goumilev. L'échec de la transition démocratique et économique sous l'ère Eltsine va fortement contribuer à la réémergence de cette idéologie. Comme l'explique Laruelle (2001, p. 72), le néoeurasisme est protéiforme. Dans la lignée d'un Aleksandr Panarin, il s'agit de défendre la notion d'empire. Il existe un courant " plus culturaliste et folkloriste " qui fait l'éloge de " l'alliance slavotürke » et " la revalorisation de l'Empire mongol et des minorités turko-musulmanes "; il y a enfin le néo-eurasisme d'Aleksandr Dugin, très en vogue dans les cercles politiques (Laruelle, 2001, p. 72). Une chose est sure : tous ces courants veulent inscrire le destin russe en Orient, en jetant aux oubliettes l'identité européenne de la Russie et les petits frères slaves (situés trop à l'Ouest). II s'agit d'une troisième voie permettant de dépasser l'opposition binaire entre slavophilie et occidentalisme. Ce n'est plus Pierre le Grand qui est adulé, mais Gengis Khan et Tamerlan. C'est dans ce contexte idéologique qu'il faut comprendre le développement de toute une série d'organisations 
régionales par la Russie avec les pays de son Étranger proche. Dans ce maillage institutionnel, les pays d'Asie centrale occupent une place essentielle. Deux organisations se distinguent. L'Union économique eurasiatique a été créée par un traité signé en 2014, initialement par la Russie, le Bélarus et le Kazakhstan, auxquels se sont joints l'Arménie et le Kirghizistan ; le Tadjikistan est candidat. Notons que cette union a signé des accords de libre-échange avec la Chine et l'Iran notamment. Et puis sur les plans politique et militaire, on retrouve l'Organisation de coopération de Shanghai établie en 2001. "L'Ouzbékistan, le Kazakhstan, le Kirghizstan et le Tadjikistan ont rejoint la Chine et la Russie au sein de la SCO (Shanghai Cooperation Organisation), considérée par certains observateurs comme un OTAN oriental constitué pour résister à l'hégémonie américaine (Lindley, 2009, p. 130). L'Inde et le Pakistan ont rejoint l'OCS en 2017. L'Iran, l'Afghanistan et la Mongolie ont le statut d'États observateurs. On notera aussi dans cette imbrication l'existence de l'Organisation du Traité de sécurité collective qui regroupe les États de l'Union économique eurasiatique plus le Tadjikistan.

Ces différentes alliances illustrent bien l'activisme russe dans l'espace eurasiatique et les puissants liens entretenus entre la Russie et les ex-républiques soviétiques d'Asie centrale (excepté le Turkménistan, le pays étant embourbé dans un régime délirant qui assis sur ses réserves de gaz, n'est pas enclin à coopérer avec qui que ce soit et n'en a pas besoin : tout le monde lui achète son gaz et le laisse tranquille). Cela soulève également la question de savoir si les pays d'Asie centrale sont acteurs ou sujets de ce «Grand Jeu ». II n'en reste pas moins que " d'une certaine manière, I'Asie centrale a donné naissance au concept d' "Eurasie informelle ». Toutes les nations et destinations du continent sont désormais interconnectées " (Lindley, 2009, p. 130). Pour ces États, les routes de la soie ne sont pas simplement un moyen de se désenclaver mais également de diversifier leurs économies notamment grâce à un développement touristique fondé sur l'imaginaire des routes. Comme Thorez (2016) l'explique :

Dans tous les pays, la rhétorique de la « Route de la soie " alimente donc le discours géopolitique, de même qu'elle nourrit le récit national - elle fait ainsi l'objet d'un traitement important dans les livres d'histoire, qui rappelle la proximité des sphères politique et scientifique dans la région. Elle est aussi valorisée dans l'espace public - à Almaty, à Bichkek ou à Tachkent, d'importantes artères ont été renommées pour porter le nom de la « Route de la Soie ». Pour les États centrasiatiques, associer la « Route de la soie " à l'Asie centrale contribue par ailleurs à façonner une représentation pittoresque de la région, qui est instrumentalisée pour inciter les touristes à venir découvrir les sociétés « authentiques » d'un Orient « éternel ». ( $p$. 303)

On voit donc bien toute la complexité que ces routes revêtent. Elles sont, toute à la fois, l'objet d'enjeux géopolitiques, géostratégiques et idéologiques de la part des grandes puissances, sujet de discours identitaires et mythes fondateurs qui relèvent d'une subjectivité étatique. 


\section{Marginalisation de l'Europe dans l'espace eurasiatique du $21 \mathrm{e}$ siècle}

On peine à discerner quelle place occupe l'Europe dans cette configuration. En dehors des nombreux jeunes Européens qui arpentent ces routes en sac à dos, il y a, de façon plus pragmatique, le programme TRACECA (Transport Corridor Europe Caucase Asie), mentionné plus haut. Lancé par I'Union européenne dès le début des années 1990, il visait lui aussi à réactiver les routes de la soie et montrait bien que les Européens avaient à cœur de jouer un rôle actif dans la région afin d'assurer leur approvisionnement en énergie sans être dépendants de la Russie. II s'agit principalement pour les Européens de fournir une assistance technique et financière pour développer un réseau multimodal (routier, maritime et ferroviaire) reprenant le tracé des différentes routes de la soie. Aujourd'hui treize États sont partenaires: les trois pays du Caucase sud (Arménie, Azerbaïdjan, Géorgie), deux pays de l'UE (Bulgarie et Roumanie), l'Ukraine et la Moldavie, l'Iran et la Turquie, et quatre pays d'Asie centrale (Kazakhstan, Kirghizstan, Ouzbékistan et Tadjikistan). Il s'agit d'un projet extrêmement ambitieux doté d'une infrastructure bureaucratique (groupes de travail, groupes d'experts, secrétariat permanent de la commission intergouvernementale, etc.) comme seuls les eurocrates peuvent le concevoir. A priori, TRACECA s'inscrit directement dans une logique concurrente au projet chinois. Cependant, le programme européen a deux désavantages comparés à son rival. Premièrement, la Chine a moins à se préoccuper de questions politiques, la situation est plus délicate pour l'UE qui, par exemple, a plus de pression pour prendre en compte des dimensions environnementales, ou des considérations liées aux droits humains. Deuxièmement, l'engagement financier fourni par l'UE s'avère complètement risible. En effet, selon les données disponibles sur le site Internet officiel de TRACECA (qui n'existe qu'en anglais et ...en russe), la liste des projets d'investissements directs terminés a couté à Bruxelles 50900000 euros (TACECA, 2019). Or, pour le projet $B R I$ " les estimations varient et chiffrent le cout de l'ensemble de ces chantiers à entre 4 000 et 26000 milliards de dollars " (Mottet et Lasserre, 2018, p. 37) que la Chine n'a pas l'intention de payer toute seule, mais ces chiffres démontrent clairement que le projet européen et le projet chinois n'ont pas du tout la même ambition. Ainsi que Mottet et Lasserre $(2018, p$. 37) le notent, le projet BRI comporte plusieurs dimensions : un volet terrestre et ferroviaire, un volet maritime, une coopération économique renforcée, une coopération énergétique et une "coopération visant à renforcer les liens entre les populations ». On est loin de l'aspect purement technique de l'Union européenne. En d'autres termes, si l'Europe avait très tôt saisi l'importance de réactiver les routes de la soie pour appuyer son statut potentiel de superpuissance dans le monde de l'après Guerre froide, elle ne s'en est pas donné les moyens. Le rapprochement et la coopération entre la Russie, la Chine, l'Iran et la Turquie (les autres pays de la région devant suivre), même s'ils ne sont que stratégiques, ont néanmoins des conséquences concrètes, au premier chef la marginalisation de l'Europe sur les plans politique, géopolitique et géostratégique.

Ce grand ensemble eurasiatique dont les routes de la soie forment la moelle épinière ont permis l'émergence de zones de contacts, d'échanges, de circulation et de biens comme nous l'avons soulevé plutôt. Mais il est également empreint de nombreuses «zones de fractures, de discontinuités, d'interfaces et de conflits dans la longue durée » comme le fait remarquer Bruneau 
(2018, p. 8). Ainsi, il est impossible aujourd'hui de comprendre les ressorts de ce qui semble s'apparenter à un génocide des Ouïgoures par les autorités chinoises sans connaître l'histoire millénaire des routes de la soie qui se développent à partir de ce Tartastan oriental (appelé Xinjiang par Pékin), foyer du peuple turcophone et musulman que sont les Ouïgoures (Lindley, 2009, p.124). Tout comme il est impossible de comprendre le " problème kurde " peuple de culture et de langue iranienne mais pratiquant un Islam sunnite, habitant les régions montagneuses représentant les marches des empires perse, ottoman et russe. Impossible encore de comprendre les conflits qui ont ressurgi ces trois dernières décennies dans les Balkans tout d'abord après l'implosion de la Yougoslavie et plus récemment dans la région de l'isthme Mer Baltique-Mer Noire. Ces deux régions de l'Europe, carrefours de religions (Islam, Judaïsme, orthodoxie, catholicisme) et d'espaces culturels et linguistiques (slave, finno-ougrien, turcophone) variés, ont été historiquement des lieux pivots où les biens (et personnes) venus des routes de la soie parvenaient à destination pour être échangés. Tous ces problèmes politiques auxquels I'Union européenne devrait aspirer à répondre - sa puissance reposant en grande partie sur son soft power diplomatique - semblent être dans les mains d'autres acteurs. L'erreur fondamentale des Européens aura été de refuser à la Turquie d'entrer dans ses rangs. En effet, la Turquie représente historiquement - et très concrètement la porte d'entrée européenne vers ce monde eurasiatique. La Turquie est également le pivot de tout le programme TRACECA (De Tapia, 2018, p. 57). L'Europe semble sans cesse hésiter lorsqu'il s'agit de ses relations avec ses voisins, proches ou plus lointains, surement en partie parce qu'elle n'a jamais su quelles étaient ses frontières; un problème identitaire qui s'est accru avec la chute de ses empires, la fin de l'époque où le monde était européen.

Paradoxalement, l'imaginaire de ces routes forme une mémoire collective commune à tous ces peuples (des Français aux Ouïgoures en passant par les Roumains, les Turkmènes ou les Égyptiens), tout en les séparant inexorablement tant la coupure Est/Ouest, Orient/Occident - aura été travaillé par les pouvoirs politiques. Car il n'y a pas de déterminisme géographique. Si ce dernier existait, les frontières entre l'Orient et l'Occident auraient été fixes, telle chaine de montagnes ou telle mer. Or ce ne fut jamais le cas. Les frontières Orient/Occident ont toujours été dynamiques répondant aux besoins et intérêts impérieux des puissances politiques. Ainsi il en est par exemple de la région du Caucase: l'Europe s'arrête-t-elle aux flancs nord des montagnes du Caucase ou au bassin hydraulique azerbaidjanais ? Tout dépend de ce que les grandes puissances veulent faire des petits États du Sud-Caucase - Arménie, Géorgie, Azerbaïdjan - coincés entre les volontés impérialistes des Russes, des Turcs, des Perses, des Anglais, et plus récemment des Américains. « À l'ouest, la mer Caspienne constitue une frontière informelle séparant l'Asie centrale du Caucase, où l'Arménie, I'Azerbaïdjan et la Géorgie, laissées à l'abandon depuis la disparition de l'URSS, n'ont de cesse de revendiquer leur statut européen. Selon les critères géographiques traditionnels, ils sont effectivement situés à l'ouest de l'Oural et à une latitude au nord de Gibraltar » (Lindley, 2009, p. 129). Ces derniers font bien partie du Conseil de l'Europe, comme la Turquie et la Russie, mais ils demeurent en dehors de I'Union européenne. Au final ces hésitations européennes font qu'aujourd'hui, l'Europe semble à l'écart des grands enjeux de l'espace eurasiatique. 


\section{Conclusion}

Certains chercheurs, comme Thorez (2016, p. 298), sont critiques de ce paradigme de la " Route de la soie " qui ne prendrait pas en compte les dynamismes et les nombreuses transformations de cette région à l'œuvre ces dernières décennies. II n'en demeure pas moins que ces routes de la soie font l'objet d'une activité discursive importante et que leur réactivation provoque des effets concrets notamment en ce qu'elle marginalise la place de l'Europe. Le problème c'est qu'au fond, l'espace eurasiatique n'existe qu'en termes de traces mémorielles pour les Européens. Fondamentalement l'Europe se situe hors du nouveau " Grand Jeu » dont les deux acteurs principaux sont la Russie et la Chine. En réalité, la grande question qui se pose comme le souligne Bruneau (2018) est la suivante : "Les deux constructions eurasiatiques à partir de l'Europe russe ou de la Chine asiatique seront-elles opposées, voire antagoniques, génératrices éventuelles de conflits, ou simplement concurrentes, pouvant devenir complémentaires et s'articuler l'une à l'autre plus ou moins harmonieusement ? " (p. 15).

\section{Références}

Arnason, J. (2000). Approaching byzantium: Identity, predicament and afterlife. Thesis Eleven, 62(1), 39-69.

Braudel, F. (1988). La Dynamique du capitalisme. Paris : Flammarion.

Bruneau, M. (2018). L'Eurasie, un impensé de la géographie : continent, empire, idéologie ou projet? L'Espace géographique, 47(1), 1-18.

Chakrabarty, D. (2000). Provincializing Europe: Postcolonial thought and historical difference. Princeton : Princeton University Press.

Delanty, G. The making of a post-western Europe: A civilizational analysis. Thesis Eleven, 72(1), 8-25.

Deleuze, G. et Guattari, F. (1980). Mille plateaux, Paris : les Éditions de Minuit.

Elias N. (2003). La Dynamique de l'Occident. Paris : Pocket.

Farchy, J. et al. (2016, 14 september). One belt, one road. Financial Times. Repéré à https://ig.ft.com/sites/special-reports/one-belt-one-road/

Fidan, H. (2010). Turkish foreign policy towards Central Asia. Journal of Balkan and Near Eastern Studies, 12(1), 109-121.

Glissant É. (1990). Poétique de la relation : Poétique III. Paris : Gallimard.

Köstem, S. (2017). When can idea entrepreneurs influence foreign policy?: Explaining the rise of the 'Turkic World' in Turkish foreign policy", Foreign Policy Analysis, 13(3), 722-740.

Laruelle, M. (2001). Le néo-eurasisme russe : L'Empire après l’Empire ? Cahiers du monde russe, 42(1), 71-94. Lindley, C. (2009). Asie centrale : le Heartland. Le Débat, 1(153), 115-130.

Mackinder, H. (1904). The geographical pivot of history. The Geographical Journal, 23(4), 421-437.

Ménard, P. et al. (2001-2008[1298]). Marco Polo, Le Devisement du monde : 6 volumes. Ménart, Genève, Droz, édition critique scientifique.

Mottet, É. et Lasserre, F. (2018). L'initiative «Belt and Road», stratégie chinoise du « Grand Jeu »? Diplomatie, 90, 36-40.

Racine, P. (2011). Marco Polo, marchand ou reporter ? Le moyen age, 117(2), 315-344.

Rezakhani, K. (2010). The road that never was: The Silk Road and Trans-Eurasian Exchange. Comparative Studies of South Asia, Africa and the Middle East, 30(3), 420-433. 
Tapia, S. (2018). Mégaprojets: Développement économique, aménagement du territoire ou megalomania turque? Moyen-Orient, 37, 54-59.

Thorez, J. (2016). La renaissance de la « Route de la soie » : un mythe qui occulte les migrations internationales. Revue européenne des migrations internationales, 32(3 et 4), 297-317.

TRACECA. (2019). Investment Projects. Repéré à http://www.traceca-org.org/en/investments/

Tranchant, M. et Ponthieux, J. (2017, 2 septembre). Quand Marco Polo rencontre Kubilaï Khan : voyage dans la Mongolie du XIIle siècle. Le Figaro. Repéré à http://www.lefigaro.fr/theatre/2017/09/02/0300320170902ARTFIG00010-quand-marco-polo-rencontre-kubilai-khan-voyage-dans-la-mongolie-du-xiiiesiecle.php

Schmidt, H. (2019, 1 mai). Qui est Nahurito, le nouvel empereur japonais ? RFI, Repéré à http://www.rfi.fr/asie-pacifique/20190501-est-naruhito-nouvel-empereur-japonais-erereiwa?ref=tw i 